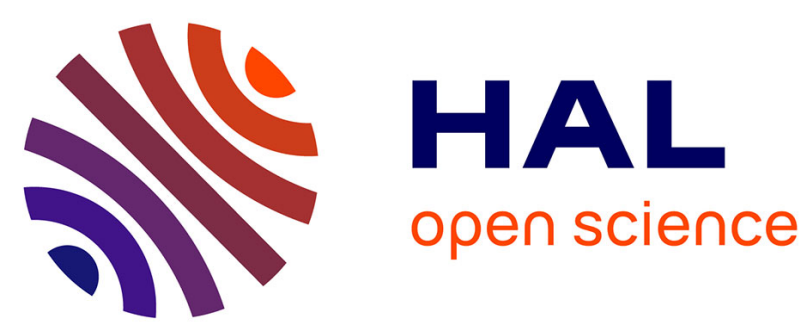

\title{
Theoretical and experimental evidence of Fano-like resonances in simple monomode photonic circuits
}

\author{
A. Mouadili, E.H. El Boudouti, A. Soltani, Abdelkrim Talbi, Abdellatif
}

Akjouj, Bahram Djafari-Rouhani

\section{- To cite this version:}

A. Mouadili, E.H. El Boudouti, A. Soltani, Abdelkrim Talbi, Abdellatif Akjouj, et al.. Theoretical and experimental evidence of Fano-like resonances in simple monomode photonic circuits. Journal of Applied Physics, 2013, 113 (16), pp.164101. 10.1063/1.4802695 . hal-00819665

\section{HAL Id: hal-00819665 https://hal.science/hal-00819665}

Submitted on 14 Sep 2021

HAL is a multi-disciplinary open access archive for the deposit and dissemination of scientific research documents, whether they are published or not. The documents may come from teaching and research institutions in France or abroad, or from public or private research centers.
L'archive ouverte pluridisciplinaire HAL, est destinée au dépôt et à la diffusion de documents scientifiques de niveau recherche, publiés ou non, émanant des établissements d'enseignement et de recherche français ou étrangers, des laboratoires publics ou privés.

\section{(c)(1)}

Distributed under a Creative Commons Attribution| 4.0 International License 


\section{Theoretical and experimental evidence of Fano-like resonances in simple monomode photonic circuits}

Cite as: J. Appl. Phys. 113, 164101 (2013); https://doi.org/10.1063/1.4802695

Submitted: 15 January 2013 . Accepted: 09 April 2013 . Published Online: 25 April 2013

A. Mouadili, E. H. El Boudouti, A. Soltani, A. Talbi, A. Akjouj, B. Djafari-Rouhani, et al.
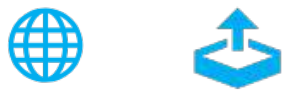

\section{ARTICLES YOU MAY BE INTERESTED IN}

Control of acoustic absorption in one-dimensional scattering by resonant scatterers

Applied Physics Letters 107, 244102 (2015); https://doi.org/10.1063/1.4938121

Y-shaped magnonic demultiplexer using induced transparency resonances

AIP Advances 9, 035011 (2019); https://doi.org/10.1063/1.5080350

Acoustically induced transparency using Fano resonant periodic arrays

Journal of Applied Physics 118, 164901 (2015); https://doi.org/10.1063/1.4934247

\section{HUDEN \\ Instruments for Advanced Science}

- Knowledge

- Experience.

- Expertise

Click to view our product catalogue

Contact Hiden Anaytica for further decails:

W www.HidenAnalytical.com B info@hiden.co.uk
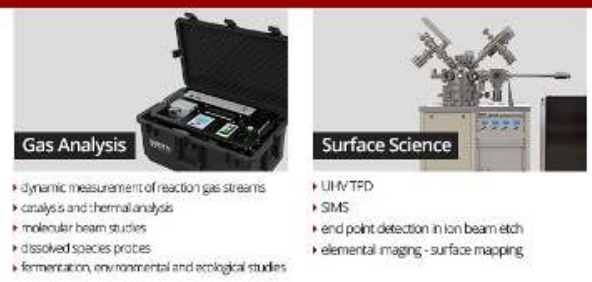

, elenertad magng-grfaxe maporg

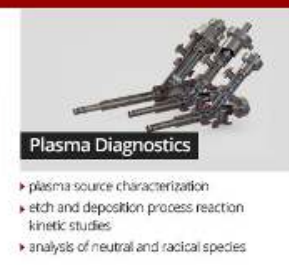

andists of nestrat and racical spestes

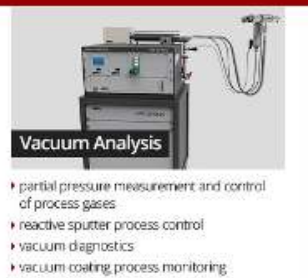
vecuuri coatrig process 


\title{
Theoretical and experimental evidence of Fano-like resonances in simple monomode photonic circuits
}

\author{
A. Mouadili, ${ }^{1}$ E. H. El Boudouti,,${ }^{1,2, a)}$ A. Soltani, ${ }^{3}$ A. Talbi, ${ }^{4}$ A. Akjouj, ${ }^{2}$ \\ and B. Djafari-Rouhani ${ }^{2}$ \\ ${ }^{1}$ Laboratoire de Dynamique et d'optique des matériaux, Département de Physique, Faculté des Sciences, \\ Université Mohamed I, Oujda, Morocco \\ ${ }^{2}$ UFR de Physique, Institut d'Electronique, de Microélectronique et de Nanotechnologie (IEMN), \\ UMR CNRS 8520, Université Lille 1 Sciences et Technologies, 59655 Villeneuve d'Ascq, France \\ ${ }^{3}$ Institut d'électronique, de Microélectronique et de Nanotechnologie (IEMN), UMR CNRS 8520, \\ Université Lille 1 Sciences et Technologies, 59655 Villeneuve d'Ascq, France \\ ${ }^{4}$ LIA LEMAC-IEMN UMR CNRS 8520, ECLille, PRES Université Lille Nord de France, \\ 59655 Villeneuve d'Ascq, France
}

(Received 15 January 2013; accepted 9 April 2013; published online 25 April 2013)

\begin{abstract}
A simple photonic device consisting of two dangling side resonators grafted at two sites on a waveguide is designed in order to obtain sharp resonant states inside the transmission gaps without introducing any defects in the structure. This results from an internal resonance of the structure when such a resonance is situated in the vicinity of a zero of transmission or placed between two zeros of transmission, the so-called Fano resonances. A general analytical expression for the transmission coefficient is given for various systems of this kind. The amplitude of the transmission is obtained following the Fano form. The full width at half maximum of the resonances as well as the asymmetric Fano parameter are discussed explicitly as function of the geometrical parameters of the system. In addition to the usual asymmetric Fano resonance, we show that this system may exhibit an electromagnetic induced transparency resonance as well as well as a particular case where such resonances collapse in the transmission coefficient. Also, we give a comparison between the phase of the determinant of the scattering matrix, the so-called Friedel phase, and the phase of the transmission amplitude. The analytical results are obtained by means of the Green's function method, whereas the experiments are carried out using coaxial cables in the radio-frequency regime. These results should have important consequences for designing integrated devices such as narrow-frequency optical or microwave filters and high-speed switches. This system is proposed as a simpler alternative to coupled-micoresonators. @ 2013 AIP Publishing LLC [http://dx.doi.org/10.1063/1.4802695]
\end{abstract}

\section{INTRODUCTION}

Often the scattering of waves in composite materials involves propagation along different paths and, as a consequence, results in interference phenomena, where constructive interference corresponds to resonant enhancement and destructive interference to resonant suppression of the transmission. This kind of resonance followed by anti-resonance is called Fano resonance ${ }^{1}$ and is characterized by an asymmetric line profile. The asymmetry originates from a close coexistence of resonant transmission and resonant reflection and can be reduced to the interaction of a discrete localized mode with a continuum of propagation modes. ${ }^{2}$ Fano was the first to describe such resonances when he was attracted by unusual sharp peaks in the absorption spectra of noble gases observed by Beutler. ${ }^{3}$ The asymmetric line shapes have been reported in the electronic transport in mesoscopic systems using the Aharonov-Bohm systems. ${ }^{4-9}$ Mainly, the subject of these studies was to use these interferometric systems to show the conditions for the existence and the collapse of Fano resonances as function of the applied current voltage and magnetic flux. These studies are also related to

\footnotetext{
a) Author to whom correspondence should be addressed. Electronic mail: elboudouti@yahoo.fr
}

the investigation of the electronic states of quantum dots ${ }^{4-9}$ as well as to the understanding ${ }^{10-13}$ of the transmission phase jumps by $\pi$ between two adjacent resonances in relation with the experiments of Yacoby et al. ${ }^{14,15}$ The analogy between scattering properties of electrons and other elementary excitations suggests that this type of feature can also appear in other vibrational systems. ${ }^{16}$

When the resonance falls between two anti-resonances, Fano resonance behaves like electromagnetic induced transparency (EIT) resonance. EIT $^{17}$ is the phenomenon that a sharp transparent window associated with steep dispersion is induced into opaque atomic media. It is due to the destructive quantum interference between different excitation pathways of the excited states. ${ }^{18}$ These systems have shown potential applications for slow light and optical data storage. ${ }^{17-21}$ Several works have demonstrated that Fano and EIT-like behaviors are not restricted to quantum systems and can be extended to classical systems, such as coupled microresonators, ${ }^{22-30}$ photonic crystal waveguides coupled to cavities, ${ }^{31-38}$ acoustic slender tube waveguides ${ }^{39-43}$ and periodic arrays of square rods, ${ }^{44}$ plasmonic nanostructures, and metamaterials. $^{45-51}$ With regard to optical resonances, microring, microdisk, and microsphere resonators ${ }^{22-30}$ coupled to a straight waveguide have shown EIT and Fano resonances with high quality factors. The resonances can be 
tuned by changing the diameters of the resonators, their separation (coupling) to the straight waveguide and the distance between the resonators.

It is worth noting that one dimensional (1D) photonic crystals made of coaxial cables have shown several interesting properties like in 2D and 3D counterpart systems. Among these properties, the existence of band gaps with or without defect modes in periodic and quasi-periodic structures, ${ }^{52-60}$ add/drop filters, ${ }^{61}$ slow and fast light, the so-called superluminal and subluminal, in periodic ${ }^{57-59}$ structures and MachZender-like ${ }^{62,63}$ interferometers. Also, recent works ${ }^{64-67}$ have shown that several properties of quantum graphs could be successfully simulated by microwave networks made of coaxial cables. These structures are attractive since their production is more feasible at any wavelength scale and they require only simple analytical and numerical calculations.

In some recent papers, ${ }^{13,39,68}$ we have proposed a new simple filter structure to study theoretically Fano resonances for acoustic, mesoscopic, and magnonic 1D circuits. This structure consists of two side stubs, which play the role of resonators, grafted at two sites on an infinite waveguide (see Fig. 1). We have shown analytically and numerically that this simple structure can exhibit transmission gaps and Fanolike resonances. In particular, we have shown that the transmission amplitude through such a system can be written following the Fano-like shape around these resonances. In addition, we have given an explicit expression of the Fano parameter $^{1}$ as well as the position and the width of the Fano resonances $^{1}$ as a function of the geometrical parameters of the system. The transmission gaps and Fano-like resonances have been established through an analysis of the transmission function (amplitude and phase) obtained within the framework of the Green's function. In addition to these quantities, the Green's function approach also enables one to deduce easily the local and total densities of states. ${ }^{52} \mathrm{~A}$ recent paper ${ }^{40}$ has confirmed experimentally these theoretical results on acoustic tubes in a more simple structure consisting a paired stubs placed at the same axial position (i.e., without introducing the segment $d_{2}$ in Fig. 1).

In this work, we give an extension of these works to study both theoretically and experimentally the possibility of existence of Fano resonances in a photonic circuit made of coaxial waveguides in the radio-frequency domain. Also, we show a comparison between the phase of the determinant of the scattering matrix, the so-called Friedel phase, and the phase of the transmission amplitude. Such a comparison has been only studied theoretically $^{10,11}$ in mesoscopic systems because of the difficulties in the measurement of the reflection coefficient. The possible realization and performance of such a

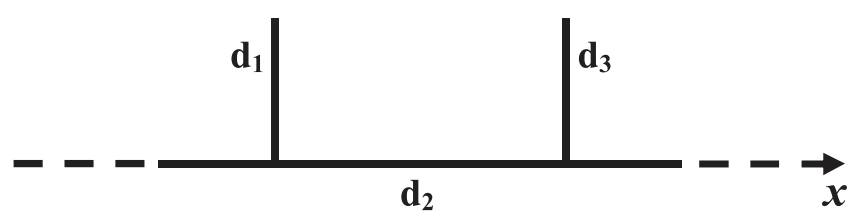

FIG. 1. Schematic illustration of the one-dimensional electromagnetic waveguide of length $d_{2}$ with dangling resonators on both sides. The whole structure is inserted between two semi-infinite waveguides. The lengths of the dangling resonators are $d_{1}$ and $d_{3}$. system in the optical range is also discussed. It is worth mentioning that a theoretical study has been performed on a similar structure made of metal-insulator-metal stub pair for application to plasmonic waveguide modulator ${ }^{45,46}$ The structure in Ref. 45 is similar to the acoustic one proposed in Ref. 40 with two different stubs grafted at the same position along the guide. However, one can show that such a structure may give only EIT-like resonance and not asymmetric Fano resonance as it is the case for the structure presented here.

This paper is organized as follows. In Sec. II, we give a brief review of the theoretical model used in this work as well as the analytical results of the structure depicted above. These results are necessary for an analytical understanding of the new phenomenon obtained for the structure proposed in this work. Section III is devoted to the symmetric Fano resonances which behave like EIT resonances, Sec. IV gives the evidence of asymmetric Fano resonances as well as the particular case where such resonances collapse in the transmission coefficient. The conclusions are presented in Sec. V.

\section{METHOD OF THEORETICAL AND NUMERICAL CALCULATIONS}

Our theoretical analysis is performed with the help of the interface response theory ${ }^{69}$ of continuous media, which allows the calculation of the Green's function of any composite material and then, total and local densities of states as well as transmission and reflection coefficients. In what follows, we give the expressions of the Green function elements for the building blocks (finite and semi-infinite wires) and for the final structure (Fig. 1).

\section{A. Inverse surface Green's functions of the elementary constituents}

We consider an infinite homogeneous isotropic dielectric wire $\mathrm{i}$ characterized by its characteristic impedance $Z_{i}$ and the relative permittivity $\varepsilon_{i}$. An electromagnetic field is propagating through the cable in the dielectric-filled space between the center conductor and shield. In the limit where the widths of the wires are much smaller than their lengths, the telegrapher's equation describing microwave networks, the socalled TEM waves, is given by

$$
\left(\frac{\partial^{2}}{\partial x^{2}}+\omega^{2} \varepsilon_{i} / c^{2}\right) E(x)=0,
$$

where $\mathrm{c}$ is the speed of light and $\omega$ is the angular frequency of the wave. Then, the corresponding Green's function between two points $\mathrm{x}$ and $\mathrm{x}^{\prime}$ of this wire is defined by ${ }^{16}$

$$
\left(\frac{\partial^{2}}{\partial x^{2}}+\omega^{2} \varepsilon_{i} / c^{2}\right) G\left(x, x^{\prime}\right)=\delta\left(x-x^{\prime}\right),
$$

whose solution is given by ${ }^{16}$

$$
G_{i}\left(x, x^{\prime}\right)=-\frac{e^{j \alpha_{i}\left|x-x^{\prime}\right|}}{2 F_{i}}
$$

where $F_{i}=-j \omega / Z_{i}, \alpha_{i}=\omega \sqrt{\varepsilon_{i}} / c$, and $j=\sqrt{-1}$. Let us recall that $\sqrt{\varepsilon_{i}}=n_{i}$ defines the index of refraction of the 
coaxial. Its inverse called the velocity factor is given by $V_{F}=\frac{1}{c \sqrt{L_{0} C}}$, where $L_{0}$ and $C$ are the inductance and the capacitance per unit length of the cable, respectively. $L_{0}$ and $C$ are frequency independent.

Before addressing the problem of the simple structure presented in this work (see Fig. 1), it is helpful to know the surface elements of its elementary constituents, namely, the Green function of a finite wire of length $d_{i}, i=1,2,3$, and of a semi-infinite wire. The boundary conditions at the ends of all the wires are $H=0$ (vanishing magnetic field). The wire of length $d_{2}$ is bounded by two surfaces located at $x=0$ and $x=d_{2}$ (Fig. 1). These surface elements can be written in the form of a $(2 \times 2)$ matrix $g_{2}(M M)$, within the interface space $M=\left\{0,+d_{2}\right\}$. The inverse of this matrix takes the following form: ${ }^{16}$

$$
g_{2}^{-1}(M M)=\left(\begin{array}{cc}
-\frac{\omega C_{2}}{Z_{2} S_{2}} & \frac{\omega}{Z_{2} S_{2}} \\
\frac{\omega}{Z_{2} S_{2}} & -\frac{\omega C_{2}}{Z_{2} S_{2}}
\end{array}\right)
$$

The inverse of the surface Green's functions of the dangling resonators grafted at the sites $\{0\}$ and $\left\{d_{2}\right\}$ is given by $g_{1}^{-1}(0,0)=-\omega S_{1} / Z_{1} C_{1}$ and $g_{3}^{-1}\left(d_{2}, d_{2}\right)=-\omega S_{3} / Z_{3} C_{3}$, where $C_{i}=\cos \left(\frac{\omega d_{i} \sqrt{\varepsilon}}{c}\right), S_{i}=\sin \left(\frac{\omega d_{i} \sqrt{\varepsilon}}{c}\right), \quad i=1,2,3$. The inverse of the surface Green's functions of the two semiinfinite waveguides surrounding the whole structure is given by $g_{s}^{-1}(0,0)=g_{s}^{-1}\left(d_{2}, d_{2}\right)=j \omega / Z_{s}$, where $Z_{s}$ is the characteristic impedance of the wire "s." In what follows, we suppose that all the wires are standard coaxial cables with the same characteristic impedances and permittivities (i.e., $Z_{1}=Z_{2}=Z_{3}=Z_{s}=Z \approx 50 \Omega$ and $\varepsilon_{1}=\varepsilon_{2}=\varepsilon_{3}=\varepsilon_{s}=\varepsilon=2.3$ ). We report on results of calculated transmission coefficients and phase or phase time as a function of frequency. Using the Green's function method, ${ }^{16}$ the expression giving the inverse of the Green's function of the whole system given in Fig. 1 can be obtained from a linear superposition of the above inverse Green's functions of the constituent, namely,

$$
g^{-1}(M M)=\frac{-\omega}{Z}\left(\begin{array}{cc}
\frac{C_{2}}{S_{2}}+\frac{C_{1}}{S_{1}}-j & -\frac{1}{S_{2}} \\
-\frac{1}{S_{2}} & \frac{C_{2}}{S_{2}}+\frac{C_{3}}{S_{3}}-j
\end{array}\right),
$$

where $C_{i}=\cos \left(\alpha d_{i}\right), S_{i}=\sin \left(\alpha d_{i}\right)$, and $\alpha=\omega \sqrt{\varepsilon} / c \quad(i=1$, $2,3)$.

\section{B. Transmission coefficient}

Let us consider an incident wave $U(x)=e^{-j \alpha x}$ launched in the left semi-infinite waveguide (Fig. 1). With the help of Eqs. (3) and (5), one easily finds the transmission wave in the right semi-infinite waveguide (Fig. 1), namely, ${ }^{16}$ $t=(2 j \omega / Z) g\left(0, d_{2}\right)$, or equivalently

$$
t=\frac{2 C_{1} C_{3}}{\chi_{1}-j \chi_{2}},
$$

where

$$
\chi_{1}=2 C_{1} C_{2} C_{3}-S_{2}\left(S_{1} C_{3}+S_{3} C_{1}\right)
$$

and

$$
\chi_{2}=2 C_{1} C_{3} S_{2}+C_{2}\left(S_{1} C_{3}+S_{3} C_{1}\right)-S_{1} S_{2} S_{3} .
$$

By the same way, the reflection coefficient is given by $r=-1+(2 j \omega / Z) g(0,0)$, or equivalently

$$
r=\frac{\chi_{1}^{\prime}+j \chi_{2}^{\prime}}{\chi_{1}-j \chi_{2}},
$$

where

$$
\chi_{1}^{\prime}=S_{2}\left(S_{1} C_{3}-S_{3} C_{1}\right)
$$

and

$$
\chi_{2}^{\prime}=C_{2}\left(S_{1} C_{3}+S_{3} C_{1}\right)-S_{1} S_{2} S_{3} .
$$

From the expression of $\mathrm{t}$ (Eq. (6)), one can deduce the transmission coefficient

$$
T=\frac{4 C_{1}^{2} C_{3}^{2}}{\chi_{1}^{2}+\chi_{2}^{2}},
$$

as well as the phase

$$
\varphi=\arctan \left(\chi_{2} / \chi_{1}\right)+\pi \Theta\left[C_{1} C_{3}\right],
$$

where $\Theta$ means the Heaviside function. From Eq. (12), one can notice that the transmission zeros are induced by the side branches (i.e., $C_{1}=0$ or $C_{3}=0$ ). These two equations give the eigenmodes of the two stubs with $H=0$ boundary conditions on both sides. When the expression $C_{1} C_{3}$ changes sign at some frequencies denoted by $\omega_{n}$, then the phase (Eq. (13)) exhibits a jump of $\pi$. Another interesting quantity is the first derivative of $\varphi$ with respect to the pulsation $\omega$, which is related to the delay time taken by the photons to traverse the structure. This quantity, called delay time, is defined by ${ }^{70}$

$$
\tau_{\varphi}=\frac{d \varphi}{d \omega}
$$

and can be written as

$$
\begin{aligned}
\tau_{\varphi}= & \frac{d}{d \omega} \arctan \left(\chi_{2} / \chi_{1}\right) \\
& +\pi \sum_{n} \operatorname{sgn}\left[\frac{d}{d \omega}\left(C_{1} C_{3}\right)_{\omega=\omega_{n}}\right] \delta\left(\omega-\omega_{n}\right),
\end{aligned}
$$

where $s g n$ means the sign function. Furthermore, the density of states (DOS) of the present composite system from which we have subtracted the DOS of the semi-infinite cable is given by ${ }^{70}$

$$
\Delta n(\omega)=\frac{1}{\pi} \frac{d}{d \omega}\left[\arctan \left(\chi_{2} / \chi_{1}\right)\right]
$$

Because of the second term in the right-hand side of Eq. (15), one can deduce that $\tau_{\varphi} \neq \pi \Delta n(\omega)$ as $\tau_{\varphi}$ (Eq. (14)) 
may exhibit $\delta$ functions at the transmission zeros that do not exist in the variation of the DOS (Eq. (16)). However, if the system does not exhibit transmission zeros, then $\Theta\left[C_{1} C_{3}\right]=0$ and $\tau_{\varphi}=\pi \Delta n(\omega)$.

From the expression of $r$ (Eq. (9)), one can deduce the reflection coefficient

$$
R=\frac{\chi_{1}^{\prime 2}+\chi_{2}^{\prime 2}}{\chi_{1}^{2}+\chi_{2}^{2}}
$$

One can show easily the conservation energy, namely

$$
R+T=1 \text {. }
$$

It should be pointed out that the validity of our results is subject to the requirement that the cross section of the waveguide being negligible compared to their length and to the propagation wavelength. The assumption of mono-mode propagation is then satisfied.

\section{EIT-LIKE RESONANCE}

\section{A. Theoretical results}

Equation (12) clearly shows that the transmission zeros are due only to the dangling resonators, these occur when $C_{1}=0$ or $C_{3}=0$ (i.e., at $\omega d_{1} \sqrt{\varepsilon} / c=\left(2 m_{1}+1\right) \pi / 2$ or $\left.\omega d_{3} \sqrt{\varepsilon} / c=\left(2 m_{2}+1\right) \pi / 2\right)$. Also, one can expect the resonance (i.e., transmission unity) induced by the segment of length $d_{2}$ around $S_{2}=0$ (i.e., at $\omega d_{2} \sqrt{\varepsilon} / c=m \pi$ ) as it can be easily shown from Eqs. (6)-(12) $\left(m, m_{1}\right.$, and $m_{2}$ are positive integers). Therefore, in order to show the possibility of existence of symmetric Fano resonance which behaves like EIT-resonance, i.e., a resonance squeezed between two transmission zeros, we have to take $d_{1}$ and $d_{3}$ slightly different from $0.5 d_{2}$. Indeed, at $\Omega=\omega d_{2} \sqrt{\varepsilon} / c=m \pi, m=1,2, \ldots$, the expression of the transmission function (Eq. (6)) becomes

$$
t= \pm \frac{2 C_{1} C_{3}}{2 C_{1} C_{3}+j \sin \left[\alpha\left(d_{1}+d_{3}\right)\right]} .
$$

In particular, if $\alpha\left(d_{1}+d_{3}\right)=m \pi, \alpha d_{1} \neq\left(m_{1}+0.5\right) \pi$, and $\alpha d_{3} \neq\left(m_{2}+0.5\right) \pi$, one obtains a resonance that reaches unity (i.e., $T=1$ ). An example corresponding to this situation is given in Fig. 2(a) where $d_{1}=0.46 d_{2}$ and $d_{3}=0.54 d_{2}$ (with $d_{1}+d_{3}=d_{2}$ ). All the curves are given with the dimensionless frequency $\Omega / \pi$ in order to discuss the Fano and EIT resonances around $\Omega / \pi=1$. One can notice that the resonance at $\Omega=\pi$ is squeezed between two zeros (indicated by solid circles on the abscissa of Fig. 2(a)) induced by the dangling resonators as it is also illustrated in the plot describing the variation of the phase (Fig. 2(c)). The width of this resonance increases as far as $d_{1}$ and $d_{3}$ deviate from $0.5 d_{2}$ (see below). In the particular case where $\alpha d_{1}=\left(m_{1}+0.5\right) \pi$ and $\alpha d_{3}$ $=\left(m_{2}+0.5\right) \pi$, the numerator and denominator of $\mathrm{t}$ (Eq. (19)) vanishes altogether. In this case, the resonance as well as the two zeros induced by the resonators fall at the same position, then the resonance collapses, the transmission coefficient vanishes and the phase drops by $\pi$ as will be shown in Sec. IV.

The resonance in Fig. 2(a) shows the same characteristics as a Fano resonance but with two zeros of transmission around the resonance instead of one as is usually the case. ${ }^{1,2}$ Such resonances behave like EIT resonances. ${ }^{17}$ Indeed, we can obtain an approximate analytical expression for the transmission function (Eq. (6)) in the vicinity of the resonance. A Taylor expansion around $\Omega=\pi$ (i.e., $\Omega=\pi+\varepsilon$ with $\varepsilon / \pi \ll 1$ ) enables us to obtain

$$
t=-\frac{\zeta \zeta^{\prime}}{\varepsilon^{2} / 2+\zeta \zeta^{\prime}+j \varepsilon\left(1-\zeta \zeta^{\prime} / 2\right)},
$$

where $\zeta=\Delta+\varepsilon\left(\frac{1}{2}+\Delta\right), \zeta^{\prime}=-\Delta+\varepsilon\left(\frac{1}{2}-\Delta\right)$, and $\Delta$ is the detuning of $d_{1}$ and $d_{3}$ from $0.5 d_{2}$ (i.e., $\Delta=\pi\left(0.5-d_{1} / d_{2}\right)$ ).

From Eq. (20), one can show that the transmission coefficient $\mathrm{T}$ can be written (following the symmetric Fano lineshape or EIT-like resonance) in the form: ${ }^{39}$

$$
T=A \frac{\left(\varepsilon+q_{1} \Gamma\right)^{2}\left(\varepsilon-q_{2} \Gamma\right)^{2}}{\varepsilon^{2}+\Gamma^{2}},
$$
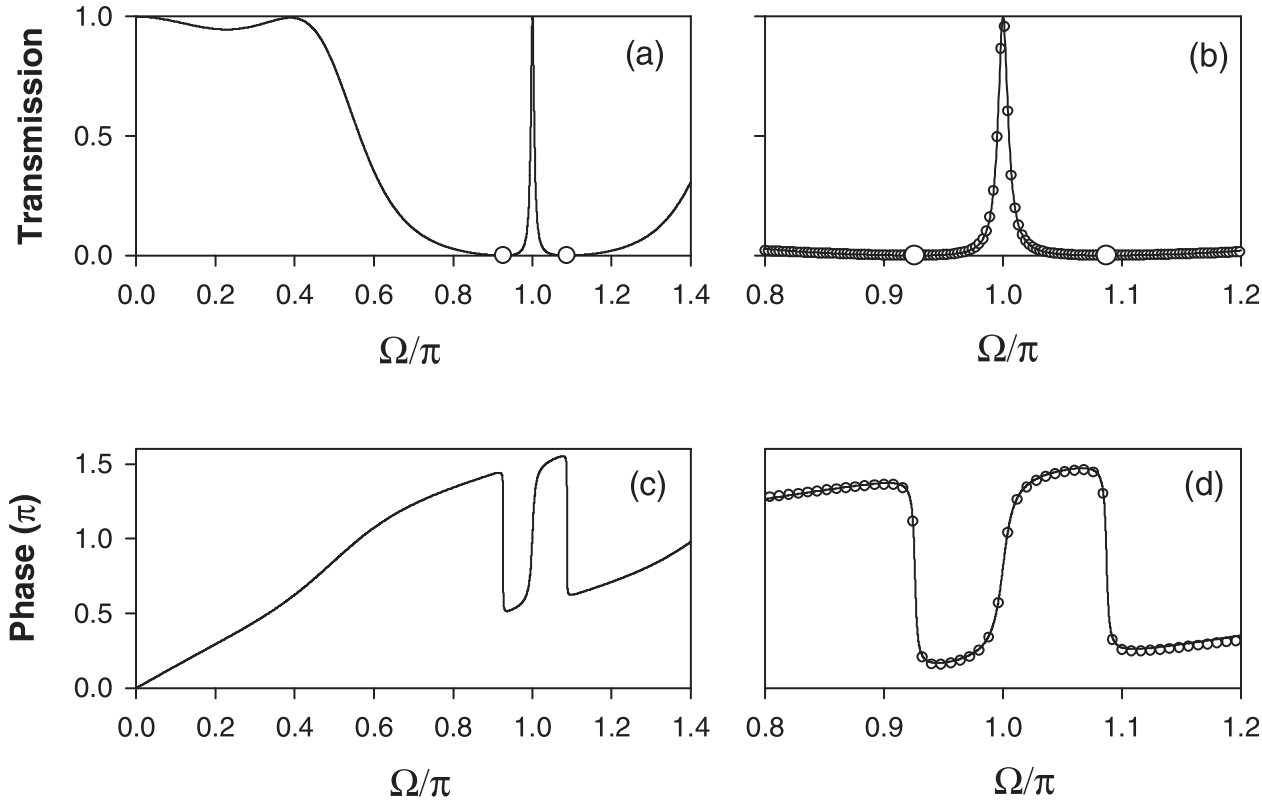

FIG. 2. (a) Theoretical (solid line) variations of the transmission coefficient versus the dimensionless frequency $\Omega$ for the structure depicted in Fig. 1. The lengths of the resonators are taken such that $d_{1}=0.46 d_{2}$ and $d_{3}=0.54 d_{2}$. White circles on the abscissa axis indicate the positions of the transmission zeros induced by the dangling resonators on both sides of the resonance. (c) Same as in (a) but for the variation of the phase. (b) and (d) compare the approximate results obtained by Taylor expansion (open circles) around the resonance with the exact calculations (solid lines). 
where $A=\frac{\left(\frac{1}{4}-\frac{\Delta^{2}}{\pi^{2}}\right)^{2}}{\left(1+\frac{\Delta^{2}}{2}\right)^{2}} . \Gamma=\frac{\Delta^{2}}{1+\Delta^{2} / 2}$ characterizes the width of the resonance falling at $\varepsilon=0$ (i.e., $\Omega=\pi$ ), $q_{1}=\frac{1+\frac{\Delta^{2}}{2}}{\Delta\left(\frac{1}{2}+\frac{\Delta}{\pi}\right)}$ and $q_{2}=\frac{1+\frac{\Delta^{2}}{2}}{\Delta\left(\frac{1}{2}-\frac{\Delta}{\pi}\right)}$ are the coupling parameters; they give qualitatively the strength of the interference between the bound state and the propagating continuum states. ${ }^{1,2}$ One can notice that when increasing $\Delta, \Gamma$ increases and $\mathrm{q}$ decreases.

The results of the approximate expression (Eq. (21)) are shown in Fig. 2(b) by open circles. These results are in accordance with the exact ones (solid lines) and clearly show that the resonance is a Fano symmetric or EIT-like resonance with $q_{1}=14.85, q_{2}=17.43$ and width $2 \Gamma=0.031 \pi$. In addition, in the periodic photonic crystals, a perturbation is often introduced to the system in order to create the resonance state in the gap. ${ }^{55-57}$ However, the above calculation shows that, without introducing any perturbation in the structure, one can find a well defined symmetric Fano resonance with a width $2 \Gamma$ and coupling parameters $q_{1}$ and $q_{2}$ that can be adjusted by tailoring the lengths of the resonators (i.e., $\Delta$, see below). Equation (20) enables us also to deduce an approximate expression for the phase as

$$
\varphi=\pi \Theta(\zeta)+\pi \Theta\left(\zeta^{\prime}\right)-\arctan \left[\varepsilon\left(1-\zeta \zeta^{\prime} / 2\right) /\left(\varepsilon^{2} / 2+\zeta \zeta^{\prime}\right)\right] .
$$

This function is plotted by open circles in Fig. 2(d) and clearly shows two abrupt phase drops of $\pi$ at $\zeta=0$ and $\zeta^{\prime}=0$ (i.e., $\varepsilon_{1}=q_{1} \Gamma$ and $\varepsilon_{2}=q_{2} \Gamma$ ) in accordance with the exact results (solid line).

The Green's function approach enables one also to deduce the local LOS (LDOS). The details of these calculations are given in Ref. 16. The LDOS reflects the behavior of the square modulus of the electric field inside the structure. An analysis of the LDOS as a function of the space position (Fig. 3) clearly shows that the EIT resonance in Fig. 2(a) is confined inside the horizontal cable and the stubs (see Fig. 3). In particular, the electric field is maximum in the middle of the finite horizontal waveguide of length $d_{2}$ and vanishes at its extremities, while it is maximum at the end of the stubs as the boundary conditions at these points are $H=0$. Therefore, these resonances could be classified as local resonances.

\section{B. Experimental results}

In what follows, we give an experimental evidence of the analytical and numerical results in Fig. 2. The coaxial

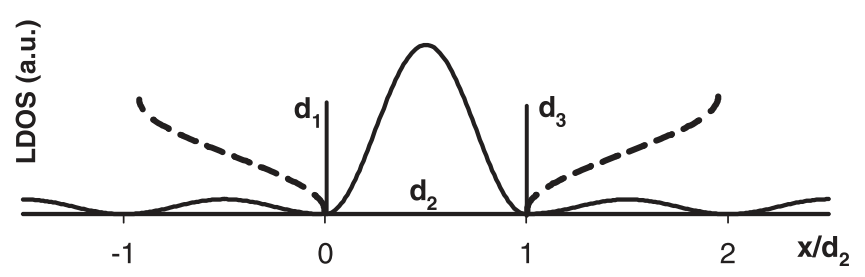

FIG. 3. The LDOS (in arbitrary units) as a function of the space positions $x / d_{2}$ and $y / d_{2}$ along the horizontal waveguide (full curve) and vertical waveguide (dashed curves), respectively, for the Fano resonance in Fig. 2(a). cable circuit studied consists of segments of RG-58/U coaxial cable of different lengths with characteristic impedance $Z=52 \Omega$. The cables were filled with polyethylene $(\varepsilon=2.3)$, corresponding to a nominal propagation speed of $0.66 \mathrm{c}$. The segments of coaxial cables were connected by standard BNC T connectors. The upper end of the stubs in Fig. 3 are open circuit (i.e., $H=0$ boundary condition). The scattering matrix $S_{n}$ of the 1D comb-like photonic crystal was measured at frequency of $1-100 \mathrm{MHz}$ with an RohdeSchwarz ZVA8 vector network analyzer 4 ports. The experimental results include the amplitude and the phase of the transmission $\left(S_{21}\right)$ and reflection $\left(S_{11}\right)$ coefficients. The attenuation inside the coaxial cables was simulated by introducing a complex dielectric constant $\varepsilon\left(\varepsilon=\varepsilon^{\prime}-j \varepsilon^{\prime \prime}\right)$. The attenuation coefficient $\alpha^{\prime \prime}$ can be expressed as $\alpha^{\prime \prime}=\varepsilon^{\prime \prime} \omega / c$. On the other hand, the attenuation specification data supplied by the manufacturer of the coaxial cables in the frequency range of $10-100 \mathrm{MHz}$ can be approximately fitted with the expression $\ln \left(\alpha^{\prime \prime}\right)=\gamma+\delta \ln (\omega)$, where $\gamma$ and $\delta$ are two constants. From this fitting procedure, a useful expression for $\varepsilon^{\prime \prime}$ as a function of frequency can be obtained under the form $\varepsilon^{\prime \prime}=0.017 f^{-0.5}$ where the frequency $f$ is expressed in Hertz.

Fig. 4(a) gives the theoretical (solid line) and experimental (open circles) variations of the transmission coefficient versus the dimensionless frequency $\Omega=\omega d_{2} \sqrt{\varepsilon} / c$ for the structure depicted in Fig. 2. The lengths of the stubs are chosen slightly different from those in Fig. 2 (i.e., $d_{1}=0.44 \mathrm{~m}, d_{3}=0.56 \mathrm{~m}$, and $\left.d_{2}=1 \mathrm{~m}\right)$. The experimental results (open circles) are very well fitted by the 1D model

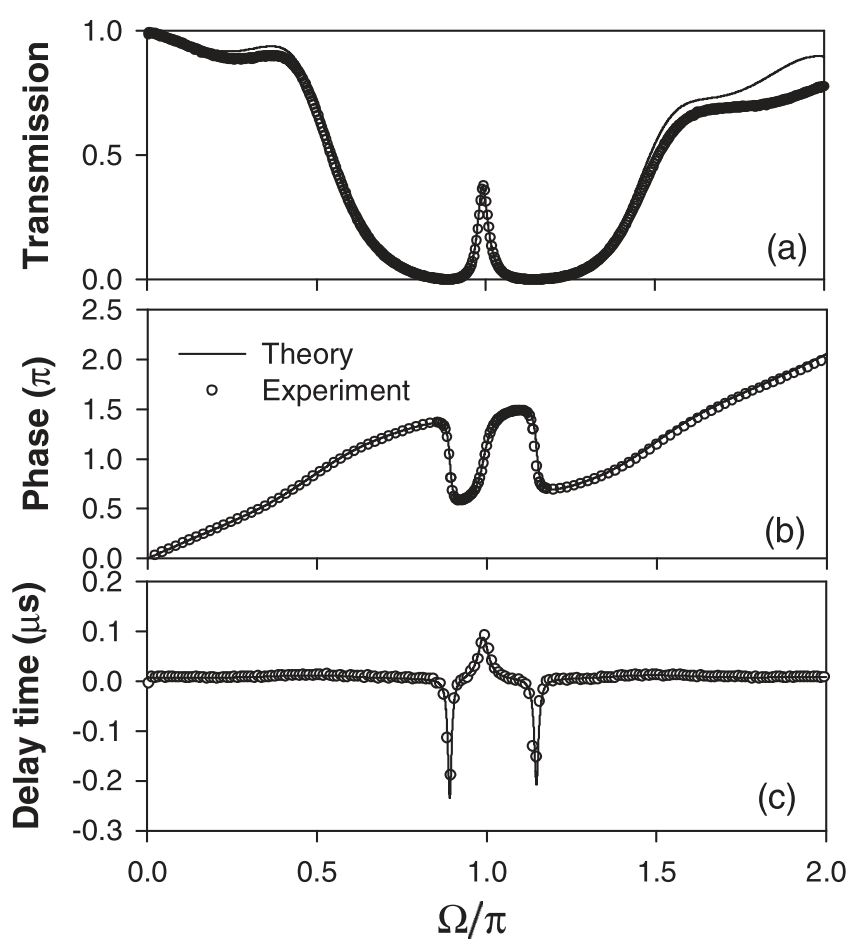

FIG. 4. (a) Theoretical (solid line) and experimental (open circles) variations of the transmission coefficient versus the dimensionless frequency $\Omega=\omega d_{2} \sqrt{\varepsilon} / c$ for the structure depicted in Fig. 1. The thicknesses of the stubs are chosen such that $d_{1}=0.44 d_{2}$ and $d_{3}=0.56 d_{2}$. (b) The same as in (a) but for the phase. (c) The same as in (a) but for the delay time. It should be noted that the domain $0<\Omega<2 \pi$ corresponds to the radio-frequency range $0<f<100 \mathrm{MHz}$. 
(solid line) using the Green's function method. One can notice that the dimensionless frequency domain $0<\Omega<2 \pi$ in Fig. 4 corresponds to the radio-frequency range $f$ such that $0<f<100 \mathrm{MHz}$. The attenuation inside the cables induces transmission depletion in Fig. 4(a) especially at high frequencies that is why the transmission does not reach unity.

One can notice that the resonance in Fig. 4(a) is well squeezed between two transmission zeros as it is illustrated in the phase (Fig. 4(b)) and the delay time (Fig. 4(c)). The EIT resonance is very well confined inside the stubs and the segment lying between them (see Fig. 3) giving rise to a large delay time in Fig. 4(c). An interesting result that can be deduced from the delay time $\tau$ (Fig. 4(c)) is the group velocity $v_{g}$ defined as the first derivative of the angular frequency $\omega$ versus the wave vector $k=\omega n(\omega) / c$ where $n(\omega)$ is the effective refractive index. Indeed, the group velocity of a pulse is the velocity at which the peak of its envelope propagates and is related to $n(\omega)$ by $^{71}$

$$
v_{g}=\frac{c}{n(\omega)+\omega \frac{d n}{d \omega}} .
$$

By regarding the system in Fig. 1 as a 1D metamaterial, the refractive index $n(\omega)$ is related to the phase $\varphi$ of the transmitted wave by the following relation:

$$
n(\omega)=\frac{\varphi c}{\omega L}
$$

where $L$ is the total effective length of the finite structure (here $L \simeq 1.5 d_{2}$ ). Substituting Eq. (24) into Eq. (23), the group velocity as a function of the effective length $L$ and delay time $\tau=d \varphi / d \omega$ can be obtained as

$$
v_{g}=L / \tau
$$

Equations (23)-(25) clearly show that in frequency regions of normal dispersion (i.e., $\frac{d n}{d \omega}>0$ ), $v_{g}$ can lead to a very small group velocity $v_{g} \ll c$ (slow light or subluminal group velocity), whereas in the case of anomalous dispersion (i.e., $\frac{d n}{d \omega}<0$ or $\frac{d \varphi}{d \omega}<0$ ) the group velocity can be larger than the speed of light in vacuum (superluminal group velocity) or even negative (pulse tunneling). The latter cases are known as fast light.

The theoretical and experimental phase function $\varphi(\omega)$ is used in Eqs. (24) and (25) to obtain the interferometer's effective index $n(\omega)$ and the expected group velocity $v_{g}(\omega)$. In the example of Fig. 4(c), negative group delay around $-0.2 \mu \mathrm{s}$ is reached giving rise to a value of $v_{g}$ as small as $-0.02 c$ at the peaks situated around $\Omega=\pi$. The idea of negative group velocity amounts to several decades with the challenge of realizing pulse advancement comparable to pulse width, with a low level of pulse distortion. This phenomenon has been found first in media made of atomic vapor cells in the presence of gain. ${ }^{72}$ In all these works, it was clearly pointed out that such superluminal behavior is not at odds with either causality or Einstein's theory of special relativity, but it exclusively results from interference between the different frequency components of the pulse in an anomalous dispersion region. ${ }^{73}$ Recently, negative group velocities have been demonstrated in a linear and passive asymmetric loop made of two or three coaxial cables $57,62,63$ where constructive and destructive interferences of the waves behave like in a Mach-Zender interferometer. The advantage of the structure presented here lies in its simplicity as well as on the fact that the Fano resonances can be tuned by varying the boundary conditions at the ends of the resonators which is not the case for the loop structures.

The above results show that the group velocity at the resonance $(\Omega=\pi)$ is very slow $\left(v_{g} \approx 0.06 c\right)$, whereas it becomes very fast around the transmission zeros. Away from the resonance and anti-resonance, the group velocity $v_{g}$ is equal to the phase velocity $v_{\varphi} \approx c / n=0.65 c$ where $\mathrm{n} \approx 1.51$ is the refractive index of the homogenous coaxial cables. Therefore, all these results confirm the slow and fast light behaviors of the EIT resonance observed in this system. In Fig. 5, we have presented another case where the lengths of the two stubs are chosen far from $0.5 d_{2}$, such as $d_{1}=0.4 \mathrm{~m}$ and $d_{3}=0.6 \mathrm{~m}$. In this case, the resonance still falls at $\Omega=\pi$ but the positions of the transmission zeros induced by the two stubs depart from $\Omega=\pi$. One can notice that the full width at half maximum of the resonance increases in accordance with the approximate expression in Eq. (21) giving rise to an intense resonance in the transmission coefficient (Fig. 5(a)).

In addition to the information that can be deduced from the amplitude and the phase of the transmission coefficient, one can also use the scattering matrix defined by

$$
S=\left(\begin{array}{ll}
r & t^{\prime} \\
t & r^{\prime}
\end{array}\right),
$$
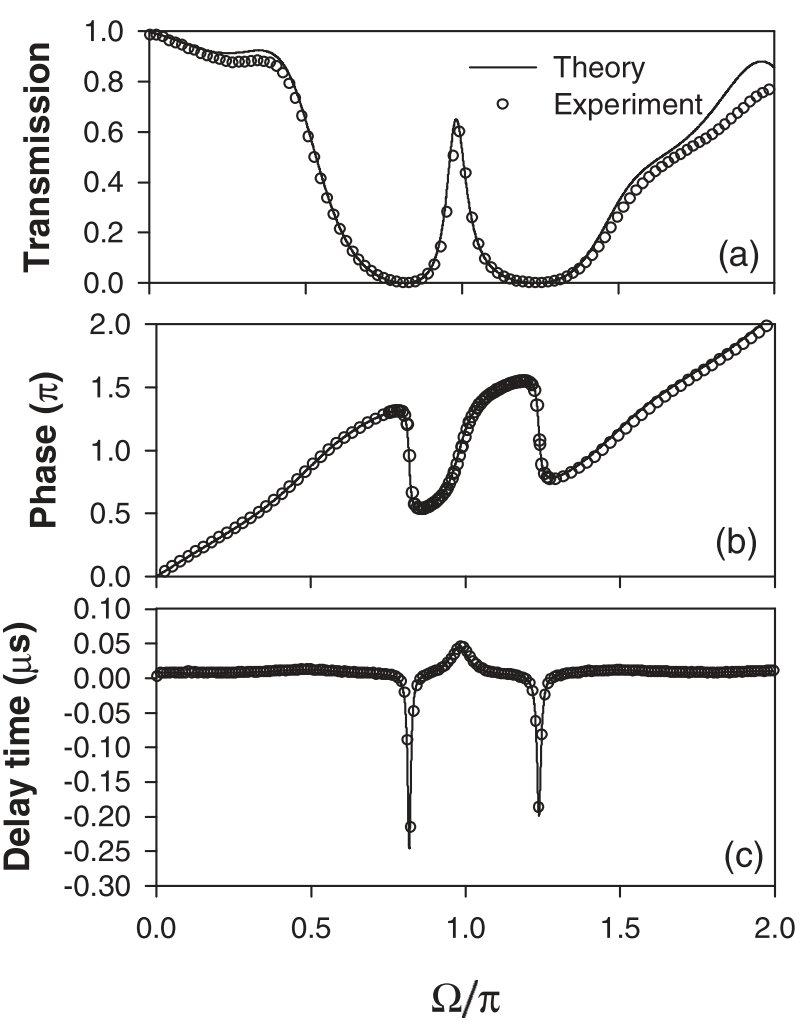

FIG. 5. (a)-(c) Same as in Figs. 4(a)-4(c) but the lengths of the resonators are taken such that $d_{1}=0.4 d_{2}$ and $d_{3}=0.6 d_{2}$. 
where $t$ and $r$ are the transmission and reflection coefficients (Eqs. (6) and (9)) for incidents waves coming from the left of the double stubs in Fig. 1. $t^{\prime}$ and $r^{\prime}$ are the transmission and reflection coefficients for incidents waves coming from the right side of the double stubs. Their expressions are obtained, respectively by Eqs. (6) and (9) by permuting the subscripts 1 and 3 (i.e., interchanging the stubs 1 and 3). One can remark here that $t=t^{\prime}$.

The Friedel sum rule relates the DOS to the scattering matrix such as ${ }^{74}$

$$
\frac{d}{d \omega} \theta_{F}(\omega)=\frac{1}{2} \frac{d}{d \omega}[\operatorname{Arg} \operatorname{Det}(S)]=\pi \Delta n(\omega),
$$

where $\theta_{F}$ is called the Friedel phase and $\Delta n(\omega)$ is the variation of the DOS or the difference in the DOS due to the presence of scatterer. Several works devoted to electronic transport in mesoscopic systems have shown theoretically ${ }^{10,11}$ the difference between the Friedel phase and phase of transmission amplitude in quasi-one-dimensional quantum wires in relation with the experiments of Yacoby et al. ${ }^{14}$ on quantum dots in the so-called Bohm-Aharonov interferometers. However, to our knowledge, such a study has not been performed yet in photonic crystals because of the difficulties in measuring both reflection and transmission coefficients. Such measurements are, however, very easy in coaxial cables. ${ }^{67}$

Figs. 6(a) and 6(b) give the amplitude and the phase of $\operatorname{Det}(\mathrm{S})$ for the structure depicted in Fig. 5. The experimental and theoretical results are plotted by open circles and solid lines, respectively. Because of the absorption in the cables, the amplitude of $\operatorname{Det}(\mathrm{S})$ is different from unity and reflects the strength of the absorption in the system in particular around the Fano resonance at $\Omega=\pi$. Contrary to the phase of the transmission amplitude (Fig. 5(b)) where phase drops
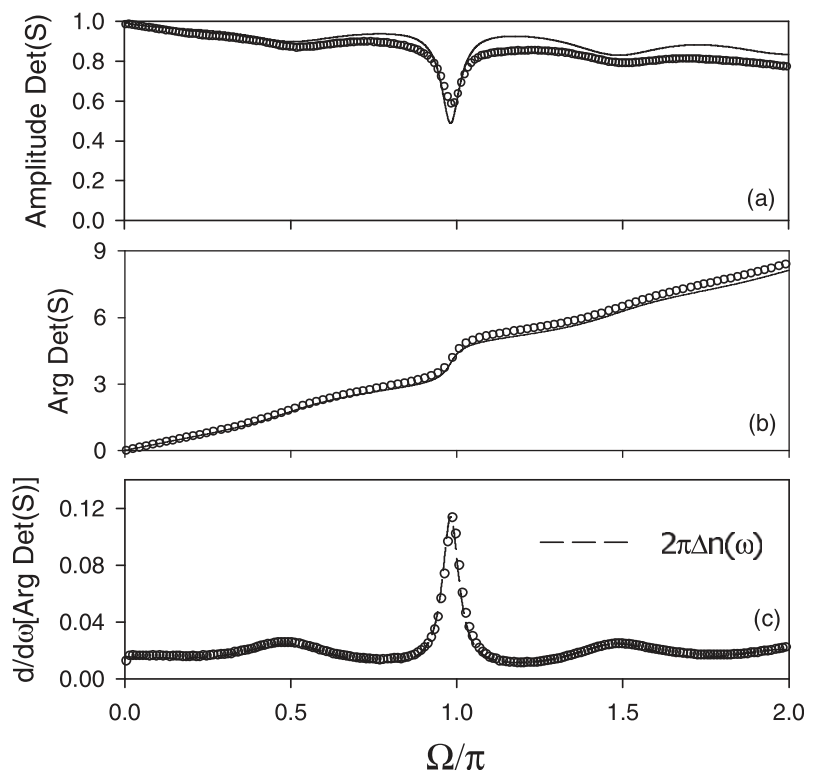

FIG. 6. (a) and (b) Variation of the amplitude and the phase (in units of $\pi$ ) of the determinant of the scattering matrix $S$ versus the dimensionless frequency $\Omega / \pi$. Open circles and solid lines correspond to experimental and theoretical results. (c) Variation of $\frac{d}{d \omega}[\operatorname{Arg} \operatorname{Det}(S)]$ (in units of $\mu$ s) obtained from (b) (open circles) and the variation of the DOS $(\Delta n(\omega))$ obtained from Eq. (16) (dashed curves). exist at the transmission zeros, the phase of $\operatorname{Det}(\mathrm{S})$ increases monotonically (Fig. 6(b)) with a fast slope around the resonance frequency, therefore, evidencing a positive resonance in its derivative versus $\omega$ (Fig. 6(c)). In Fig. 6(c), we have also plotted (dashed curves) the variation of the DOS $(\Delta n(\omega))$ given by Eq. (16). A very good agreement between $\Delta n(\omega)$ and $\frac{1}{2} \frac{d}{d \omega}[\operatorname{Arg} \operatorname{Det}(S)]$ is obtained (Eq. (27)). These results are in accordance with those found in single channel quantum scattering systems ${ }^{10,11,75}$ and give an experimental demonstration of Eqs. (15) and (16), namely, the variation of the DOS (Fig. 6(c)) is the same as the transmission delay time (Fig. 5(c)) except at the transmission zeros where the delay time exhibits additional negative delta peaks.

\section{ASYMMETRIC FANO-LIKE RESONANCE}

The structure studied in this work (Fig. 1) can exhibit also an asymmetric Fano resonance by adjusting the transmission zeros on only one side of the resonance, this can be obtained by considering a structure where the resonators are supposed to be identical with lengths slightly different from $0.5 d_{2}$. This is shown in Fig. 7(a) for $d_{1}=d_{3}=0.56 d_{2}$. Indeed, an analytical Taylor expansion around $\Omega=\pi$ enables us to write the transmission function (Eq. (6)) as

$$
t=\frac{-2 \eta^{2}}{(j+\eta)(\varepsilon+2 \eta-j \eta \varepsilon)}
$$

where $\eta=\Delta+\varepsilon\left(\frac{1}{2}+\frac{\Delta}{\pi}\right)$ and $\Delta$ is the detuning of the lengths of the two resonators from $0.5 d_{2}$ (i.e., $\Delta=\pi\left(d_{1} / d_{2}-0.5\right)$ ).

From the expression of $\mathrm{t}$ (Eq. (28)), one can deduce the following Fano line shape transmission coefficient:

$$
T=B \frac{1}{1+\eta^{2}} \frac{\left(\varepsilon-\varepsilon_{R}+q \Gamma\right)^{4}}{\left(\varepsilon-\varepsilon_{R}\right)^{2}+\Gamma^{2}} \simeq B \frac{\left(\varepsilon-\varepsilon_{R}+q \Gamma\right)^{4}}{\left(\varepsilon-\varepsilon_{R}\right)^{2}+\Gamma^{2}},
$$

where $B=\frac{\left(\frac{1}{2}+\frac{\Delta}{\pi}\right)^{4}}{\left(1+\frac{\Delta}{\pi}\right)^{2}}, \Gamma=\frac{1}{4} \frac{\Delta^{2}\left(1-\frac{\Delta}{\pi}\right)}{\left(1+\frac{\Delta}{\pi}\right)^{2}}$, and $\varepsilon_{R}=\frac{-\Delta}{1+\frac{\Delta}{\pi}}$ characterize the width and the shift of the resonance, respectively, whereas $q=\frac{2\left(1+\frac{\Delta}{\pi}\right)}{\Delta\left(\frac{1}{2}+\frac{\Delta}{\pi}\right)\left(1-\frac{\Delta}{\pi}\right)}$ is the Fano parameter.

One can notice that the resonance shifts slightly from $\Omega=\pi$ and its width is small as compared to the preceding case (Fig. 2(a)). Also q increases when $\Delta$ decreases and tends to infinity when $\Delta$ vanishes. In this case, the resonance falls at $\varepsilon_{R}=0$ and, as expected, its width $2 \Gamma$ reduces to zero (see below). The results of the approximate expression (Eq. (29)) are sketched (open circles) in Fig. 7(b) for $\Delta=\pi\left(d_{1} / d_{2}-0.5\right)=0.06 \pi$ (i.e., $d_{1} / d_{2}=0.56$ ). These results are in accordance with the exact ones (solid lines) and clearly show that the resonance presents an asymmetrical Fano shape with $|q| \simeq 21.38$ and width $2 \Gamma \simeq 0.015$. Concerning the evolution of the phase of the transmitted waves in this structure, one can notice from Eq. (4) that the numerator of the transmission function $\mathrm{t}$ vanishes when $C_{1}=C_{3}=0$ (or equivalently $\eta=0$ in the approximate result (Eq. (28)) at $\Omega=0.893 \pi$ indicated by a filled circle on the abscissa of Fig. 7(a). The transmission zeros induced by the two identical resonators fall at the same frequency, therefore, the phase (Figs. 7(c) and 7(d)) shows a phase 


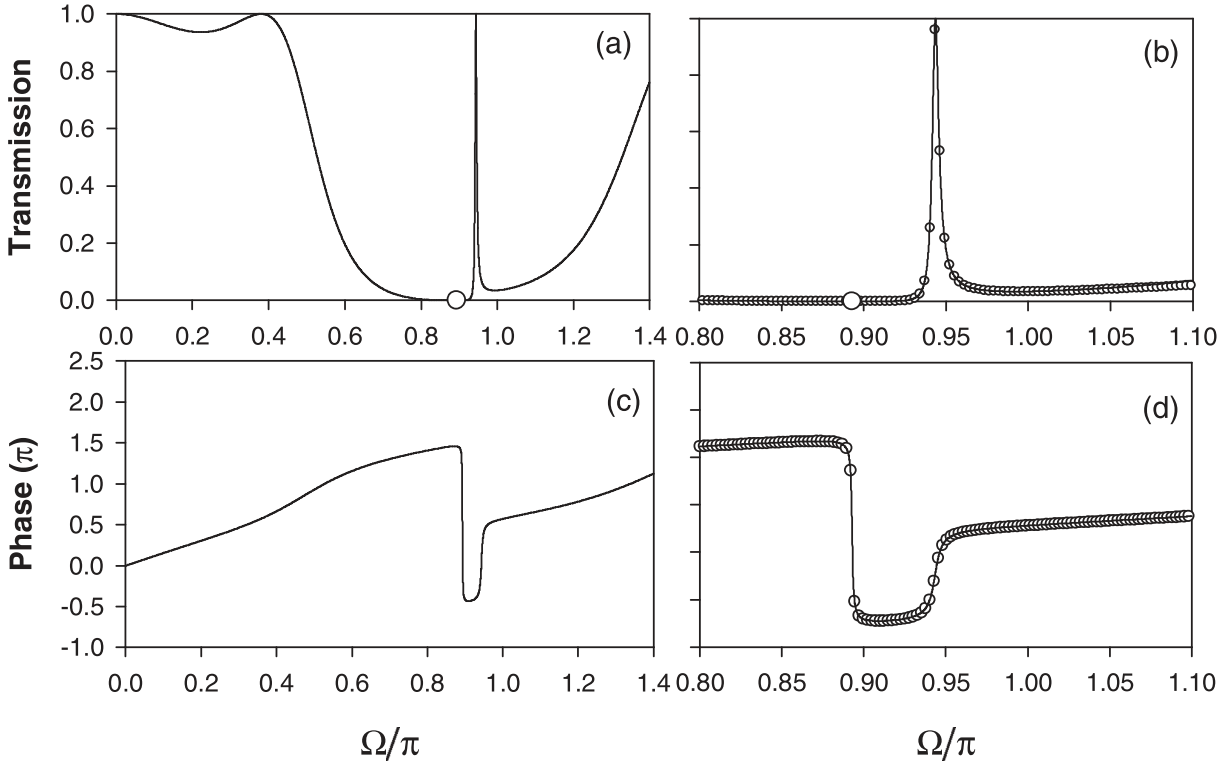

FIG. 7. (a) Theoretical (solid line) variations of the transmission coefficient versus the dimensionless frequency $\Omega$ for the structure depicted in Fig. 1. The lengths of the resonators are taken such that $d_{1}=d_{3}=0.56 d_{2}$. White circles on the abscissa axis indicate the positions of the transmission zeros induced by the dangling resonators on both sides of the resonance. (c) Same as in (a) but for the variation of the phase. (b) and (d) compare the approximate results obtained by Taylor expansion (open circles) around the resonance with the exact calculations (solid lines). drop of $2 \pi$ at these frequencies. Indeed, as the phase is defined modulo $2 \pi$, the $2 \pi$ phase change can be observed if we take into account the absorption in the system which is the case for the coaxial cables studied here.

An experimental evidence of these theoretical results is given in Fig. 8 , where $d_{1} \simeq d_{3} \simeq 0.6 \mathrm{~m}$ (i.e., $d_{1}=0.605 d_{2}$ and $d_{3}=0.625 d_{2}$ ). The experimental results (open circles) are in agreement with the numerical simulation (solid lines). One can notice here also that the intensity of the resonance does not reach unity because of the attenuation inside the cables. However, the main features of the asymmetric Fano

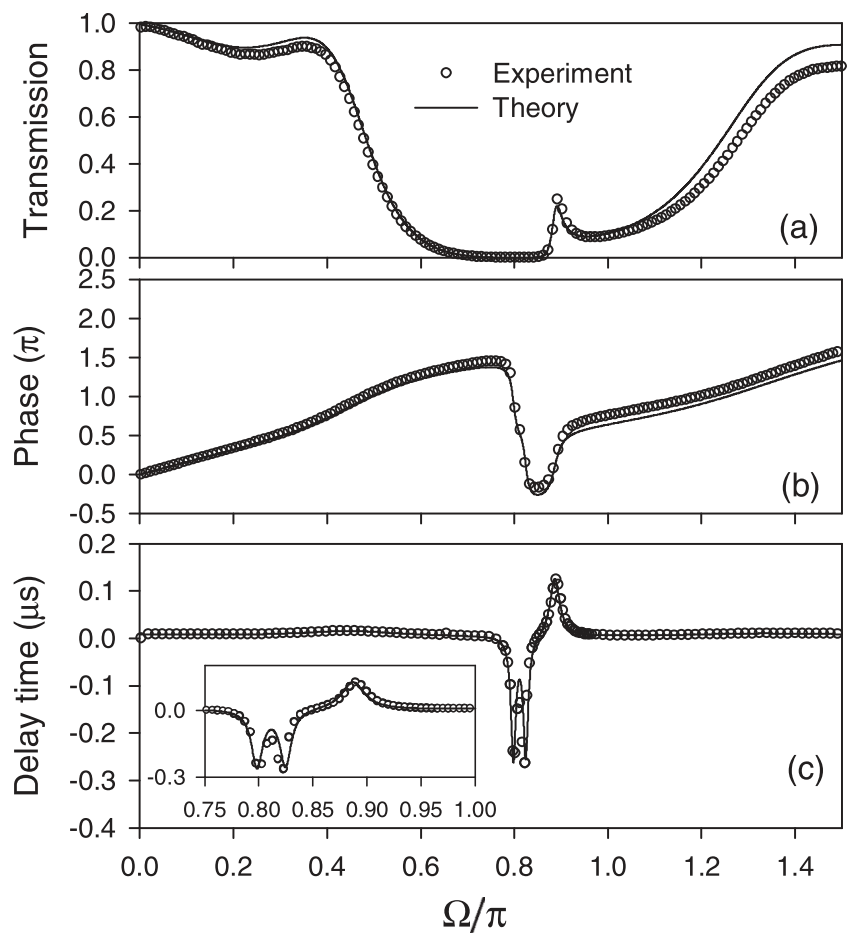

FIG. 8. (a)-(c) Same as in Figs. 5(a)-5(c) but the lengths of the resonators are taken such that $d_{1}=0.605 d_{2}$ and $d_{3}=0.625 d_{2}$ (i.e., $d_{1} \simeq d_{3}$ ). The inset in Fig. 8(c) gives an enlargement of the delay time in the frequency domain $0.75<\Omega / \pi<1$. resonance can be observed. The two resonators give rise to a phase drop of $2 \pi$ as it is illustrated in Fig. 8(b). However, because of the small difference between the lengths of $d_{1}$ and $d_{3}$, the two transmission zeros induced by the two resonators do not fall exactly at the same frequency as it can be seen in Fig. 8(c) (see also the inset) where two negative delta peaks appear in the delay time around the transmission zeros at $\Omega=0.846 \pi$ and $\Omega=0.85 \pi$.

In the particular case where $d_{1}=d_{3}=0.5 d_{2}=0.5 \mathrm{~m}$, the frequency of the Fano resonance coincides exactly with the transmission zeros induced by the two stubs at $\Omega=\pi$ (see Eqs. (21) and (29)). The width of the resonance $\Gamma$ vanishes and therefore the resonance collapses. This result is clearly demonstrated in Fig. 9(a) where a well defined dip is observed around $\Omega=\pi$. However, because of the existence of two antiresonances and one resonance at $\Omega=\pi$, the phase exhibits a jump of $\pi$ (Fig. 9(b)) giving rise to a negative delta peak in the delay time (Fig. 9(c)). This result gives a clear signature of the existence of the so-called Ghost-Fano ${ }^{76,77}$ resonance in the transmission amplitude (Fig. 9(a)). To our knowledge, this is a first experimental evidence showing the existence of such resonances with infinite lifetime.

In order to give a better insight about the profile of the Fano resonances as function of the parameter $\Delta$ (or equivalently $d_{1} / d_{2}$ ), Fig. 10(a) gives theoretical results of the characteristic features of the resonances around $d_{1} / d_{2}=0.5$ for an asymmetric resonance. On can notice that the position of the resonance decreases as function of $d_{1} / d_{2}$, its asymmetric Fano profile becomes symmetric and changes sign for $d_{1} / d_{2} \simeq 0.5$. In other words, the parameter $q$ responsible for the asymmetric Fano profile of the resonance diverges and changes sign around $d_{1} / d_{2}=0.5$. The width of the resonance decreases when $d_{1} / d_{2}$ tends to 0.5 and vanishes when $d_{1} / d_{2}$ is exactly equal 0.5 giving rise to the collapse of the resonance (Fig. 10(a)). These results are well illustrated by the plots of the approximate expressions of $\varepsilon_{R}$ (Fig. 10(b)) (or equivalently $\Omega_{R}=\pi+\varepsilon_{R}$ ), $\Gamma$ (Fig. 10(c)) and $q$ (Fig. $10(\mathrm{~d})$ ) around $d_{1} / d_{2}=0.5$. Let us notice that the reverse of the asymmetry of the lineshape of Fano resonances has been 


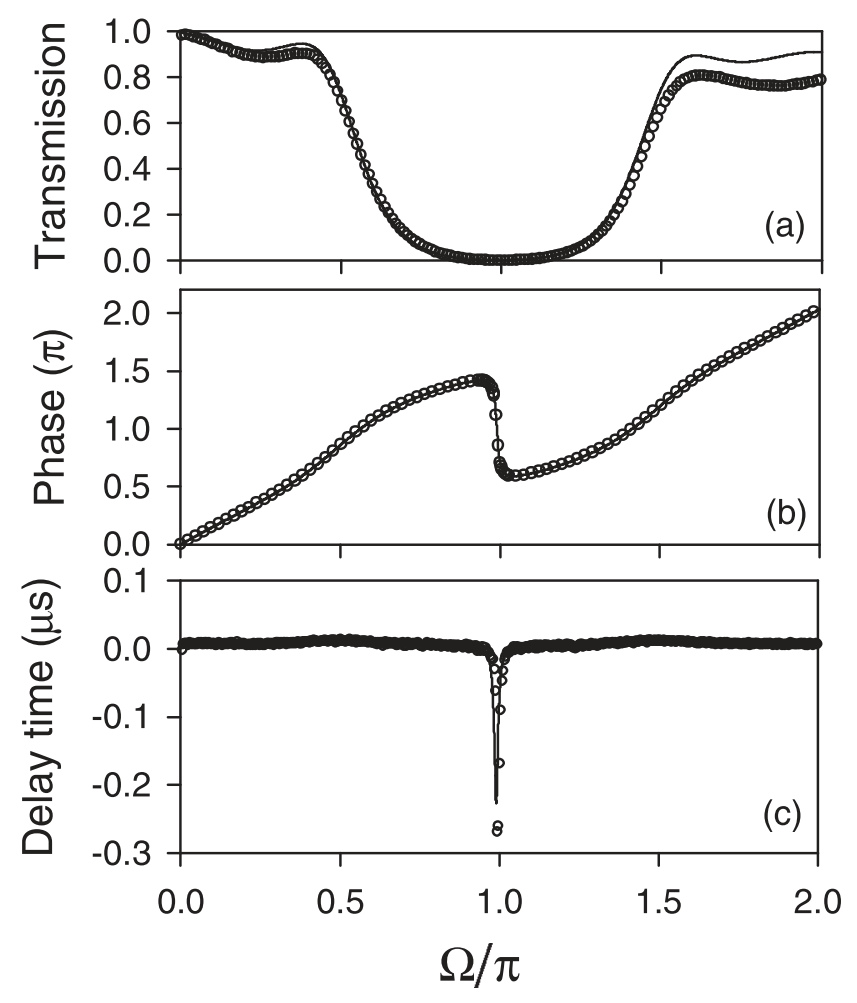

FIG. 9. (a) Theoretical (solid line) and experimental (open circles) variations of the transmission coefficient versus the dimensionless frequency $\Omega=\omega d_{2} \sqrt{\varepsilon} / c$ for the structure depicted in Fig. 1. The thicknesses of the stubs are chosen such that $d_{1}=d_{3}=0.5 d_{2}=0.5 \mathrm{~m}$. (b) The same as in (a) but for the phase. (c) The same as in (a) but for the delay time.

discussed in photonic crystal slabs by tuning the parameters of the structure, or the angle of incidence. ${ }^{34,35}$

\section{SUMMARY AND CONCLUSION}

In summary, we have clearly demonstrated that a simple geometry of an electromagnetic waveguide with dangling side resonators on both sides can pave the way to the obtention of gaps in the radio frequency domain. The existence of the stop bands in the spectrum is attributed to the zero of transmission associated with the dangling resonators (Fig. 9). The width of the transmission gaps can be increased by grafting several side resonators at each node, for instance by coupling the waveguide to side resonators on both sides (not shown here). Besides the transmission gaps, we have shown the existence of different kinds of resonances like EIT and asymmetric Fano resonances. These resonances lie at the vicinity of a transmission zero or are squeezed between two transmission zeros and collapse when they coincide with the transmission zeros. The Fano resonances are obtained by tailoring the lengths of the different branches constituting the structure. A study of the phase of the transmission function enables us to deduce several properties on the wave propagation through such structures as the delay times (or the group velocities) and the density of states. For the system studied here, the phase of the amplitude transmission exhibits phase drops at the transmission zeros, whereas the Friedel phase (or equivalently the DOS) increases monotonically with a fast slope around the resonance frequency. The resonant modes give rise to well defined peaks in the delay time and
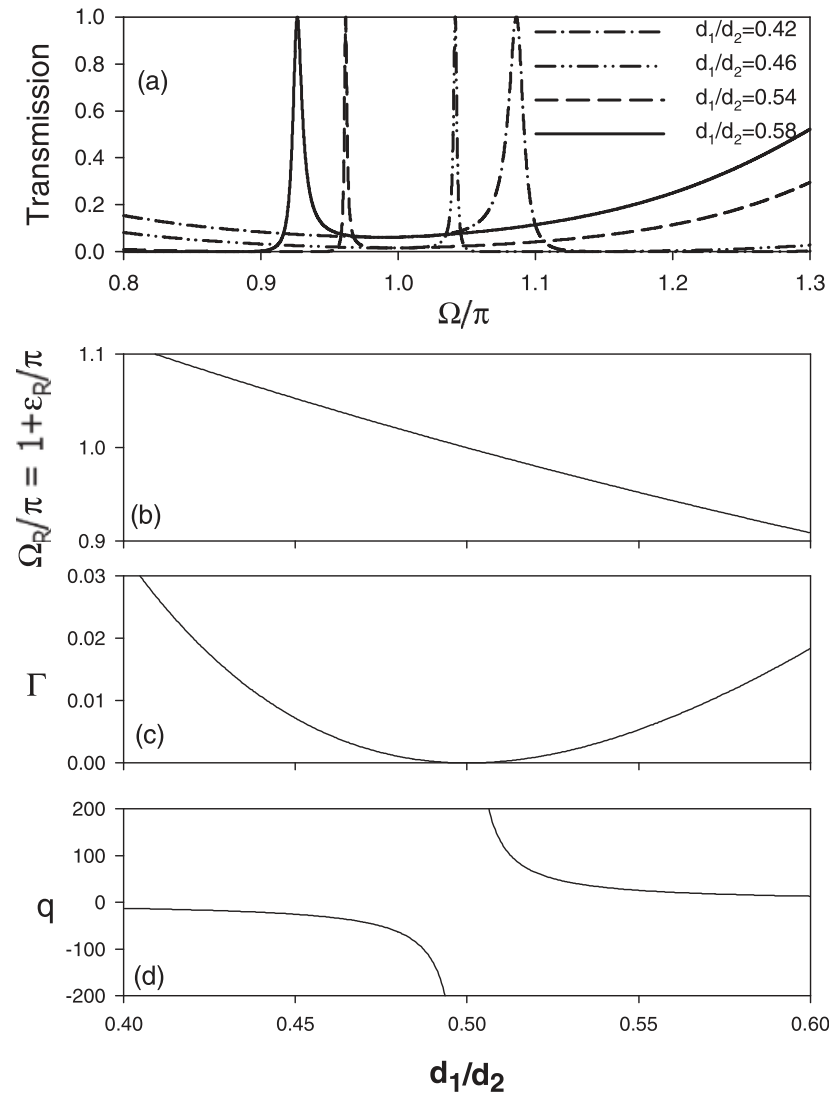

FIG. 10. Theoretical (solid line) variations of the transmission coefficient versus the dimensionless frequency $\Omega$ for different values of $d_{1} / d_{2}$ in the structure depicted in Fig. 1 with $d_{1}=d_{3}$. (b)-(d) Variations of the quantities $\varepsilon_{R}(\mathrm{~b}), \Gamma(\mathrm{c})$, and $q(\mathrm{~d})$ as a function of $d_{1} / d_{2}$ around $d_{1} / d_{2}=0.5$. The corresponding expressions are given after Eq. (29). Let us notice that the approximate expression of the position of the resonance in Fig. 10(a) is given by $\Omega_{R}=\pi+\varepsilon_{R}$.

the DOS. The theoretical results are confirmed experimentally by using standard coaxial cables connected with $\mathrm{T}$ connectors.

The advantage of the simple electromagnetic waveguide model presented in this work consists in finding simple analytical expressions that enable us to discuss the existence of Fano resonances as well as the effect of the different stubs lengths in tailoring these resonances without incorporating a defect as it is the case in periodic structures. ${ }^{55-57}$ We believe that this paper brings a new piece of work in the field of electromagnetic wave transport in $1 \mathrm{D}$ waveguide structures. This system is proposed as a simpler alternative to coupledmicroresonators.

It is worth noting that all the results presented here for the amplitude and the phase of the transmission are given versus a dimensionless frequency $\Omega=\omega d_{2} \sqrt{\varepsilon} / c$ in the range $0<\Omega<2 \pi$ which correspondence approximately to the frequency domain $0<f<100 \mathrm{MHz}$ as $d_{2}=1 \mathrm{~m}$ and $\varepsilon=2.3$. Therefore, these results remain valid in microwave and optical ranges by taking properly the thicknesses of the three finite waveguides in Fig. 1. Also, similar results to those presented here can be obtained by inserting a segment between two asymmetric loops (instead of two stubs) which play the role of resonators, however, the advantage of the structure with stubs lies in its simplicity as well as on the 
fact that the boundary conditions at the end of the stubs can be tuned to create for example an on/off switching in channel add-drop filter structures. ${ }^{78}$ Finally, let us mention that coupling several resonators with gradual lengths analogous to the so-called side-coupled integrated spaced sequence of resonators (SCISSOR) can serve to design high performance add/drop filters, multiplexers/demultiplexers, and routers. ${ }^{79}$ This work is in progress.

\section{ACKNOWLEDGMENTS}

E. H. El Boudouti gratefully acknowledge the hospitality of the Institut d'Electronique, de Microélectronique et de Nanotechnologie (IEMN), UMR CNRS 8520 and UFR de Physique, Université des Sciences et Technologies de Lille.

${ }^{1}$ U. Fano, Phys. Rev. 124, 1866 (1961).

${ }^{2}$ For a review, see A. E. Miroshnichenko, S. Flach, and Y. S. Kivshar, Rev. Mod. Phys. 82, 2257 (2010).

${ }^{3}$ H. Beutler, Z. Phys. A 93, 177 (1935).

${ }^{4}$ D. Goldhaber-Gordon, H. Shtrikman, D. Mahalu, D. Abush-Magder, U. Meirav, and M. A. Kastner, Nature (London) 391, 156 (1998).

${ }^{5}$ J. Gores, D. Goldhaber-Gordon, S. Heemeyer, M. A. Kastner, H. Shtrikman, D. Mahalu, and U. Meirav, Phys. Rev. B 62, 2188 (2000).

${ }^{6}$ Y. Ji, M. Heiblum, and H. Shtrikman, Phys. Rev. Lett. 88, 076601 (2002).

${ }^{7}$ K. Kobayashi, H. Aikawa, S. Katsumoto, and Y. Iye, Phys. Rev. Lett. 88, 256806 (2002)

${ }^{8}$ J. Kim, J.-R. Kim, J.-O. Lee, J. W. Park, H. M. So, N. Kim, K. Kang, K.-H. Yoo, and J.-J. Kim, Phys. Rev. Lett. 90, 166403 (2003).

${ }^{9}$ B. Babic and C. Schönenberger, Phys. Rev. B 70, 195408 (2004).

${ }^{10}$ T. Taniguchi and M. Buttiker, Phys. Rev. B 60, 13814 (1999).

${ }^{11}$ H. W. Lee, Phys. Rev. Lett. 82, 2358 (1999).

${ }^{12}$ J. Wu, B. L. Gu, H. Chen, W. Duan, and Y. Kawazoe, Phys. Rev. Lett. 80, 1952 (1998).

${ }^{13}$ H. Al-Wahsh, E. H. El Boudouti, B. Djafari-Rouhani, A. Akjouj, and L. Dobrzynski, Phys. Rev. B 75, 125313 (2007).

${ }^{14}$ A. Yacoby, M. Heiblum, D. Mahalu, and H. Shtrikman, Phys. Rev. Lett. 74, 4047 (1995).

${ }^{15}$ R. Schuster, E. Buks, M. Heiblum, D. Mahalu, V. Umansky, and H. Shtrikman, Nature (London) 385, 417 (1997).

${ }^{16}$ J. O. Vasseur, A. Akjouj, L. Dobrzynski, B. Djafari- Rouhani, and E. H. El Boudouti, Surf. Sci. Rep. 54, 1 (2004).

${ }^{17}$ For a review, see M. Fleischhauer1, A. Imamoglu, and J. P. Marangos, Rev. Mod. Phys. 77, 633 (2005).

${ }^{18}$ S. E. Harris, Phys. Today 50(7), 36 (1997).

${ }^{19}$ L. V. Hau, S. E. Harris, Z. Dutton, and C. H. Behroozi, Nature 397, 594 (1999).

${ }^{20}$ C. Liu, Z. Dutton, C. H. Behroozi, and L. V. Hau, Nature 409, 490 (2001).

${ }^{21}$ For a review, see I. Novikova, R. L. Walsworth, and Y. Xiao, Laser Photonics Rev. 6, 333 (2012), and references therein.

${ }^{22}$ L. Maleki, A. B. Matsko, A. A. Savchenkov, and V. S. Ilchenko, Opt. Lett. 29, 626 (2004).

${ }^{23}$ D. D. Smith, H. Chang, K. A. Fuller, A. T. Rosenberger, and R. W. Boyd, Phys. Rev. A 69, 063804 (2004).

${ }^{24}$ Q. Xu, S. Sandhu, M. L. Povinelli, J. Shakya, S. Fan, and M. Lipson, Phys. Rev. Lett. 96, 123901 (2006).

${ }^{25}$ K. Totsuka, N. Kobayashi, and M. Tomita, Phys. Rev. Lett. 98, 213904 (2007).

${ }^{26}$ M. Tomita, K. Totsuka, R. Hanamura, and T. Matsumoto, J. Opt. Soc. Am. B 26, 813 (2009).

${ }^{27}$ C. H. Raymond Ooi and C. H. Kam, Phys. Rev. B 81, 195119 (2010).

${ }^{28}$ Y.-F. Xiao, M. Li, Y.-C. Liu, Y. Li, X. Sun, and Q. Gong, Phys. Rev. A 82, 065804 (2010).

${ }^{29}$ B.-B. Li, Y.-F. Xiao, C.-L. Zou, X.-F. Jiang, Y.-C. Liu, F.-W. Sun, Y. Li, and Q. Gong, Appl. Phys. Lett. 100, 021108 (2012).

${ }^{30}$ W. Ding, B. Luk'yanchuk, and C.-W. Qiu, Phys. Rev. A 85, 025806 (2012).

${ }^{31}$ S. Fan and J. D. Joannopoulos, Phys. Rev. B 65, 235112 (2002).
${ }^{32}$ M. F. Yanik, W. Suh, Z. Wang, and S. Fan, Phys. Rev. Lett. 93, 233903 (2004).

${ }^{33}$ X. Yang, M. Yu, D.-L. Kwong, and C. W. Wong, Phys. Rev. Lett. 102, 173902 (2009).

${ }^{34}$ M. Galli, S. L. Portalupi, M. Belotti, L. C. Andreani, L. ÓFaolain, and T. F. Krauss, Appl. Phys. Lett. 94, 071101 (2009).

${ }^{35} \mathrm{Lj}$. Babíc and M. J. A. de Dood, Opt. Express 18, 26569 (2010).

${ }^{36}$ J. Zhou, D. Mu, J. Yang, W. Han, and X. Di, Opt. Express 19, 4856 (2011).

${ }^{37}$ Y. Huo, S. Sandhu, J. Pan, N. Stuhrmann, M. L. Povinelli, J. M. Kahn, J. S. Harris, M. M. Fejer, and S. Fan, Opt. Lett. 36, 1482 (2011).

${ }^{38}$ Y. Sato, Y. Tanaka, J. Upham, Y. Takahashi, T. Asano, and S. Noda, Nat. Photonics 6, 56 (2012).

${ }^{39}$ E. H. El Boudouti, T. Mrabti, H. Al-Wahsh, B. Djafari-Rouhani, A. Akjouj, and L. Dobrzynski, J. Phys.: Condens. Matter 20, 255212 (2008).

${ }^{40}$ A. Santillán and S. I. Bozhevolnyi, Phys. Rev. B 84, 064304 (2011).

${ }^{41}$ W. Tan, C. Z. Yang, H. S. Liu, Z. G. Wang, H. Q. Lin, and H. Chen, EPL 97, 24003 (2012).

${ }^{42}$ S. Hein, W. Koch, and L. Nannen, J. Fluid Mech. 664, 238 (2010); 692, 257 (2012).

${ }^{43}$ J. Fey and W. M. Robertson, J. Appl. Phys. 109, 114903 (2011).

${ }^{44}$ F. Liu, M. Ke, A. Zhang, W. Wen, J. Shi, Z. Liu, and P. Sheng, Phys. Rev. E 82, 026601 (2010).

${ }^{45}$ X. Piao, S. Yu, and N. Park, Opt. Express 20, 18994 (2012).

${ }^{46} \mathrm{G}$. Wang, H. Lu, and X. Liu, Opt. Express 20, 20902 (2012).

${ }^{47}$ N. Liu, L. Langguth, T. Weiss, J. Kästel, M. Fleischhauer, T. Pfau, and H. Giessen, Nature Mater. 8, 758 (2009).

${ }^{48}$ B. Luk'yanchuk, N. I. Zheludev, S. A. Maier, N. J. Halas, P. Nordlander, H. Giessen, and C. T. Chong, Nature Mater. 9, 707 (2010).

${ }^{49}$ J. Wu, B. Jin, J. Wan, L. Liang, Y. Zhang, T. Jia, C. Cao, L. Kang, W. Xu, J. Chen, and P. Wu, Appl. Phys. Lett. 99, 161113 (2011).

${ }^{50}$ R. Singh, I. A. I. Al-Naib, Y. Yang, D. R. Chowdhury, W. Cao, C. Rockstuhl, T. Ozaki, R. Morandotti, and W. Zhang, Appl. Phys. Lett. 99, 201107 (2011).

${ }^{51}$ X. Piao, S. Yu, S. Koo, K. Lee, and N. Park, Opt. Express 19, 10907 (2011).

${ }^{52}$ Y. El Hassouani, H. Aynaou, E. H. El Boudouti, B. Djafari-Rouhani, A. Akjouj, and V. R. Velasco, Phys. Rev. B 74, 035314 (2006).

${ }^{53}$ B. Djafari-Rouhani, E. H. El Boudouti, A. Akjouj, L. Dobrzynski, J. O. Vasseur, A. Mir, N. Fettouhi, and J. Zemmouri, Vacuum 63, 177 (2001).

${ }^{54}$ L. Poirier and A. Haché, Appl. Phys. Lett. 78, 2626 (2001).

${ }^{55}$ G. J. Schneider, S. Hanna, J. L. Davis, and G. H. Watson, J. Appl. Phys. 90, 2642 (2001).

${ }^{56}$ J. O. Vasseur, B. Djafari-Rouhani, L. Dobrzynski, A. Akjouj, and J. Zemmouri, Phys. Rev. B 59, 13446 (1999).

${ }^{57}$ E. H. El Boudouti, N. Fettouhi, A. Akjouj, B. Djafari-Rouhani, A. Mir, J. O. Vasseur, L. Dobrzynski, and J. Zemmouri, J. Appl. Phys. 95, 1102 (2004).

${ }^{58}$ A. Haché and L. Poirier, Phys. Rev. E 65, 036608 (2002); J. N. Munday and W. M. Robertson, Appl. Phys. Lett. 81, 2127 (2002).

${ }^{59}$ H. Aynaou, E. H. El Boudouti, Y. El Hassouani, A. Akjouj, B. DjafariRouhani, J. Vasseur, A. Benomar, and V. R. Velasco, Phys. Rev. E 72, 056601 (2005).

${ }^{60}$ E. H. El Boudouti, Y. El Hassouani, B. Djafari-Rouhani, and H. Aynaou, Phys. Rev. E 76, 026607 (2007).

${ }^{61}$ L. Dobrzynski, B. Djafari-Rouhani, A. Akjouj, J. O. Vasseur, and J. Zemmouri, Phys. Rev. B 60, 10628 (1999).

${ }^{62}$ M. M. Sánchez-López, A. Sánchez-Meroño, J. Arias, J. A. Davis, and I. Moreno, Appl. Phys. Lett. 93, 074102 (2008).

${ }^{63}$ J. Arias, A. Sánchez-Meroño, M. M. Sánchez-López, and I. Moreno, Phys. Rev. A 85, 033815 (2012).

${ }^{64}$ O. Hul, S. Bauch, P. Pakonski, N. Savytskyy, K. Zyczkowski, and L. Sirko, Phys. Rev. E 69, 056205 (2004).

${ }^{65}$ M. Ławniczak, O. Hul, S. Bauch, P. Seba, and L. Sirko, Phys. Rev. E 77, 056210 (2008).

${ }^{66}$ M. Ławniczak, S. Bauch, O. Hul, and L. Sirko, Phys. Rev. E 81, 046204 (2010).

${ }^{67}$ O. Hul, M. Ławniczak, S. Bauch, A. Sawicki, M. Kuś, and L. Sirko, Phys. Rev. Lett. 109, 040402 (2012).

${ }^{68}$ H. Al-Wahsh, E. H. El Boudouti, B. Djafari-Rouhani, A. Akjouj, T. Mrabti, and L. Dobrzynski, Phys. Rev. B 78, 075401 (2008).

${ }^{69}$ L. Dobrzynski, Surf. Sci. Rep. 11, 139 (1990). 
${ }^{70}$ M. L. H. Lahlaouti, A. Akjouj, B. Djafari-Rouhani, L. Dobrzynski, M. Hammouchi, E. H. El Boudouti, A. Nougaoui, and B. Kharbouch, Phys. Rev. B 63, 035312 (2001).

${ }^{71}$ L. Brillouin, Wave Propagation and Group Velocity (Academic, New York, 1960).

${ }^{72}$ L. J. Wang, A. Kusmich, and A. Dogarin, Nature (London) 406, 277 (2000).

${ }^{73}$ B. Macke and B. Segard, Eur. Phys. J. D 23, 125 (2003).

${ }^{74}$ J. Friedel, Philos. Mag. 43, 153 (1952).
${ }^{75}$ S. Souma and A. Suzuki, Phys. Rev. B 65, 115307 (2002).

${ }^{76}$ M. L. Ladrón de Guevara, F. Claro, and P. A. Orellana, Phys. Rev. B 67, 195335 (2003).

${ }^{77}$ H. Barkay, E. Narevicius, and N. Moiseyev, Phys. Rev. B 67, 045322 (2003).

${ }^{78}$ S. Fan, P. R. Villeneuve, J. D. Joannopoulos, and H. A. Haus, Phys. Rev. B 64, 245302 (2001).

${ }^{79}$ M. Mancinelli, R. Guider, P. Bettotti, M. Masi, M. R. Vanacharla, and L. Pavesi, Opt. Express 19, 12227 (2011). 